\title{
An HCC Aggressiveness Index and Blood GTP, Bilirubin and Platelet Levels
}

Brian I Carr ${ }^{*}$, Vito Guerra ${ }^{2}$, Edoardo G. Giannini ${ }^{3}$, Fabio Farinati ${ }^{4}$, Francesca Ciccarese ${ }^{5}$, Gian Ludovico Rapaccini ${ }^{6}$, Maria Di Marco ${ }^{7}$, Luisa Benvegnù ${ }^{8}$, Marco Zoli ${ }^{9}$, Franco Borzio ${ }^{10}$, Eugenio Caturelli ${ }^{11}$, Alberto Masotto ${ }^{12}$ and Franco Trevisani ${ }^{13}$

${ }^{1}$ Izmir Biomedicine and Genome Center, Dokuz Eylul University, Izmir, Turkey

${ }^{2}$ Department of Clinical Trials and Epidemiology, IRCCS de Bellis, Castellana Grotte, Italy

${ }^{3}$ Departiment of Internal Medicine, Gastroenterology Unit, University of Genoa, Italy

${ }^{4}$ Departiment of Surgical Science and Gastroenterology, Gastroenterology Unit, University of Padua, Italy

${ }^{5}$ Division of Surgery, Policlinico San Marco, Zingonia, Italy

${ }^{6}$ Internal Medicine and Gastroenterology Unit, Catholic University of Rome, Italy

${ }^{7}$ Division of Medicine, Azienda Ospedaliera Bolognini, Seriate, Italy

${ }^{8}$ Departiment of Clinical and Experimental Medicine, Medical Unit, University of Padua, Italy

${ }^{9}$ Department of Medical and Surgical Science, Internal Medicine Unit, Alma Mater Studiorum, University of Bologna, Italy

${ }^{10}$ Department of Medicine, Internal Medicine and Hepatology Unit, Ospedale Fatebenefratelli, Milan, Italy

${ }^{11}$ Gastroenterology Unit, Ospedale Belcolle, Viterbo, Italy

${ }^{12}$ Gastroenterology Unit, Ospedale Sacro Cuore Don Calabria, Negrar, Italy

${ }^{13}$ Department of Medical Surgical Sciences, Medical Semiotics Unit, Alma Mater Studiorum, University of Bologna, Italy

*Corresponding author: Brian I Carr, Visiting Professor, Program for Targeted Experimental Therapeutics, Izmir Biomedicine and Genome Center, Dokuz Eylul University, Izmir, Turkey, Tel: 1412980 4518; E-mail: brianicarr@hotmail.com

Rec date: May 17, 2016; Acc date: June 14, 2016; Pub date: June 20, 2016.

Copyright: (C) 2016 Carr Bl, et al. This is an open-access article distributed under the terms of the Creative Commons Attribution License, which permits unrestricted use, distribution, and reproduction in any medium, provided the original author and source are credited.

\begin{abstract}
Four HCC characteristics typically inform tumor behavior: maximum tumor size, number of nodules, portal vein thrombosis and serum AFP level. The sum of these parameters was recently published as an HCC Aggressiveness Index. We aimed to validate this index retrospectively in a larger and independent HCC cohort of 2706 Italian HCC patients, and to evaluate a possible relationship between the index and liver function parameters. The scores in the HCC Aggressiveness Index were again found to significantly relate to patient survival. Furthermore, in a multiple logistic regression model of the Aggressiveness Index score categories, there were significant differences in several liver parameter terciles amongst the score categories, suggesting a relationship of liver function to tumor aggressiveness. It was concluded that a prognostically significant Tumor Aggressiveness Index was validated and was found to be related to levels of some common liver function parameters.
\end{abstract}

Keywords: HCC; Aggressiveness index; Survival; Liver function

\section{Abbreviations}

HCC: Hepatocellular Carcinoma; AFP: Alpha-Fetoprotein; GGTP: Gamma Glutamyl Transpeptidase; ALKP: Alkaline Phosphatase; AST: Aspartate Aminotransferase; PVT: Portal Vein Thrombosis; MTD: Maximum Tumor Diameter; CT: Computerized Axial Tomography Scans.

\section{Introduction}

Four clinical HCC characteristics are generally considered in assessing an individual patient's tumor-related management and prognosis. They are: maximum tumor diameter (MTD), number of tumor nodules, portal vein invasion (PVT) and blood alphafetoprotein (AFP) levels (as well as presence or absence of metastasis, as with most solid tumor types). A recent 'HCC Aggressiveness' scoring system was recently described, which incorporated all 4 of these parameters and related them to survival [1]. These 4 tumor characteristics also were related to liver function parameters, given that many tumors, including HCC, arise on the basis of a chronically inflamed organ [2]. Furthermore, HCC patients with elevated bilirubin levels have also been shown to have larger tumors and increased incidence of PVT [3]. A recently validated index of systemic inflammation, using the levels of only 2 liver-derived proteins, Creactive protein (CRP) and albumin has been shown in multiple studies to be an independent prognosticator of survival [4-6]. The platelet to lymphocyte ratio is another recently described, independent inflammatory prognostic index for HCC [7]. In this paper, we validated the new Aggressiveness Index on a separate and much larger HCC patient cohort in relation to survival and we examined liver function parameters in relation to the index. We found values for the index also related significantly to survival, as well as to several liver function parameters.

\section{Methods}

\section{Patients and data collection}

Data collection: We retrospectively analyzed prospectively-collected data in the Italian Liver Cancer (ITA.LI.CA) study group database of 2706 HCC patients accrued till 2008 at 11 centers [3] who had full baseline tumor parameter data, including CT scan information on 
maximum tumor diameter (MTD), number of tumor nodules and presence of PVT and plasma AFP levels; blood counts (hemoglobin, white cells, platelets, prothrombin time); routine blood liver function tests, (total bilirubin, AST, ALKP, GGTP, albumin); demographics and survival information. ITA.LI.CA database management conforms to Italian legislation on privacy and this study conforms to the ethical guidelines of the Declaration of Helsinki. Approval for this retrospective study on de-identified patients was obtained by the Institutional Review Board of participating centers.

Aggressiveness index: To construct an index that was the expression of tumor aggressiveness, we have considered the following four parameters: Maximum Tumor dimension (MTD), alpha-fetoprotein (AFP), Portal Vein Thrombosis (PVT), and the number of tumor nodules.

The Index score of Aggression was obtained as follows: MTD (in tertiles): MTD < 4.5; $4.5 \leq$ MTD $\leq 9.6$; MTD > 9.6; scores 1, 2, 3 respectively. AFP (cut-off): AFP $<100 ; 100 \leq$ AFP $\leq 1000$; AFP $>1000$ ng/ml; scores 1, 2, 3 respectively. PVT (No/Yes): PVT (No); PVT (Yes); scores 1, 3 respectively. Tumor Nodule (number): Nodules $\leq 3$; Nodules > 3; scores 1, 3 respectively. Therefore the Aggressiveness Index was obtained as the sum of the scores for these 4 parameters (Table 1).

\begin{tabular}{|l|l|l|l|}
\hline \multirow{2}{*}{} & \multicolumn{2}{l|}{ Score } \\
\cline { 2 - 4 } & 1 & 2 & 3 \\
\hline MTD (in terciles) $(\mathrm{cm})$ & $<4.5$ & $4.5-9.6$ & $>9.6$ \\
\hline AFP (cut-off) $(\mathrm{ng} / \mathrm{mL})$ & $<100$ & $100-1000$ & $>1000$ \\
\hline PVT (No/Yes) & No & -- & Yes \\
\hline Tumor Nodules (number) & $\leq 3$ & -- & $>3$ \\
\hline
\end{tabular}

MTD: Maximum Tumor Diameter, AFP: Alpha-fetoprotein, PVT: Portal Vein Thrombosis. The Aggressiveness Index is the sum of the scores for MTD + AFP + PVT + \# Nodules.

Table 1: Aggressiveness Index is the sum of parameter scores.

Thus: MTD (in tertiles): MTD $<4.5 ; 4.5 \leq$ MTD $\leq 9.6$; MTD > 9.6; scores 1, 2, 3 respectively. AFP (cut-off): AFP $<100 ; 100 \leq$ AFP $\leq 1000$; AFP > $1000 \mathrm{ng} / \mathrm{ml}$; scores 1, 2, 3 respectively. PVT (No/Yes): PVT (No); PVT (Yes); scores 1, 3 respectively. Tumor Nodule (number): Nodules $\leq 3$; Nodules $>3$; scores 1, 3 respectively. The Aggressiveness Index score was divided into three categories for Cox analysis (Table 2): a, score $=4$; b, $4<$ score $\leq 7$; and c, score $\geq 8$.

Statistical analysis: Mean and SD for continuous variables were used as indices of centrality and dispersion of the distribution. For nonnormally distributed values we was necessary used a non-parametric methods, the Wilcoxon rank-sum (Mann-Whitney) test, for continuous variables, to test the comparisons between the Aggressiveness Index categories of liver function parameters.

The Cox proportional hazards model was applied to evaluate the predictive factors as categories of Aggressiveness Index score associated with overall survival. The results were presented as Hazard Ratio (HR) with 95\% C.I. Unconditional multiple logistic regression model was used to evaluate the Odds-Ratio of the Aggressiveness
Index score $(\geq 8)$ on the terciles of each serum variable, Gamma Glutamyl Transpeptidase (GGTP), Alkaline Phosphatase (ALKP), and Aspartate Aminotransferase (AST), total bilirubin and platelets as terciles; while albumin levels were dichotomized. All variables were included together in the model. The results were presented as OddsRatio (OR) with $95 \%$ C.I.

In all models, Cox regression and Logistic regression, the HR and the OR respectively, represent the risk for one-unit variation of the predictor variable considered as dummy variables. Patient survival between the three categories of Aggressiveness Index score was estimated with the Kaplan-Meier method and comparison of survival was made with the Breslow (generalized Wilcoxon) test. The Breslow test was used, as opposed to the log rank test, due to the large proportion of patients who died early.

When testing the hypothesis of significant association, $\mathrm{p}$-value was $<0.05$, two tailed for all analyses. Statistical analysis was performed with State Corp 2007 State Statistical Software: release 10. College Station, TX: StataCorp LP.

\section{Result}

\section{Aggressiveness index and survival}

An Aggressiveness Index was constructed as described previously [1] and as shown in Table 1, was the sum of the scores for MTD, tumor multimodality, PVT and AFP values. Using the designated cutoffs for point systems, patients were divided into 3 groups $\mathrm{a}, \mathrm{b}$ and $\mathrm{c}$ (Table 1), using a score from 4 (lowest score, with 1 point per parameter) to 12 (highest score, with 3 points per parameter). The Kaplan-Meier survival graph (Figure 1).

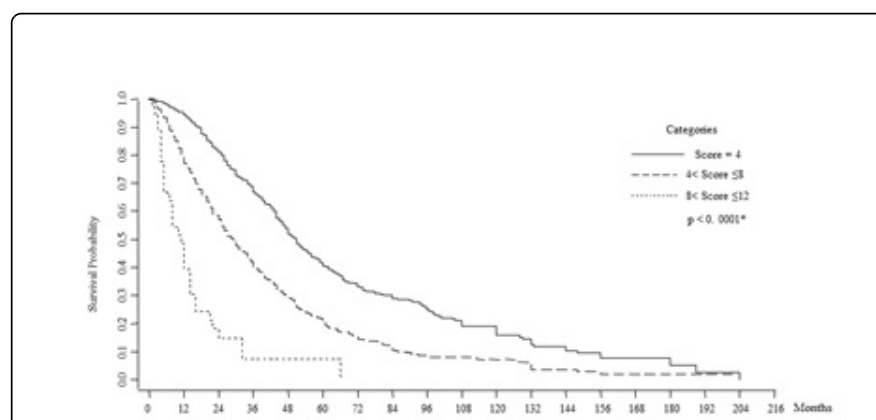

Figure 1: Kaplan-Meier Survival plots between categories of Aggressiveness Index, in patients having bilirubin $<1.5 \mathrm{mg} / \mathrm{dL}$.

Showed statistically significant differences in survival between the 3 groups. The percent survival probabilities for the 3 groups, a, b and $c$ were: $94 \%, 77 \%$ and $39 \%$ at 1 year, and $81 \%, 57 \%$ and $14 \%$ at 2 years, and $67 \%, 39 \%$ and $7 \%$ at 3 years, for the 3 groups, from the lowest to the highest scores.

A Cox proportional hazard model for death was then calculated (Table 2), with score (a) being the reference category. The hazard ratios (HRs) for groups a, b and c were: 1, 1.94 and 6.64, respectively, with pvalues of $<0.001$ for a vs. $b$, a vs. $c$ and $b$ vs. c. 
Citation: Carr BI, Guerra V, Giannini EG, Farinati F, Ciccarese F, et al. (2016) An HCC Aggressiveness Index and Blood GTP, Bilirubin and Platelet Levels. J Integr Oncol 5: 172. doi:10.4172/2329-6771.1000172

Page 3 of 6

\begin{tabular}{|c|c|c|c|c|c|c|c|}
\hline & \multicolumn{2}{|c|}{$\mathrm{HR}^{*}$} & $\mathrm{se}(\mathrm{HR})$ & $\mathbf{p}$ & 95\% C.I. & Comparisons of HRs & $\mathbf{p}$ \\
\hline \multicolumn{8}{|l|}{ Aggressiveness Index } \\
\hline Score $=4$ [Ref. category $]$ & (a) & 1 & -- & -- & -- & (a) vs (b) & $<0.001$ \\
\hline $4<$ Score $\leq 8$ & (b) & 1.94 & 0.14 & $<0.001$ & 1.68 to 2.25 & (a) vs (c) & $<0.001$ \\
\hline $8<$ Score $\leq 12$ & (c) & 6.64 & 1.27 & $<0.001$ & 4.56 to 9.66 & (b) vs (c) & $<0.001$ \\
\hline
\end{tabular}

Table 2: Cox proportional hazard model in HCC patients for death, on Aggressiveness Index Score categories in the total cohort.

\section{Aggressiveness index and liver function parameters}

The relationship between the Aggressiveness Index and liver function parameters was then examined. Firstly, the mean liver function parameters for the worst Index group (score $\geq 8$ ) were compared to the other 2 groups combined (score $<8$ ) (Table 3 ).

\begin{tabular}{|l|l|l|l|}
\hline \multicolumn{2}{|l|}{ Aggressiveness Index Score } & \\
\hline$(\mathrm{M} \pm \mathrm{SD})$ & Score $<8$ & Score $\geq 8$ & $\mathrm{p}^{\circ}$ \\
\hline GGTP $(\mathrm{IU} / \mathrm{mL})$ & $77.0 \pm 103.3$ & $96.7 \pm 87.5$ & $<0.0001$ \\
\hline ALKP $(\mathrm{IU} / \mathrm{mL})$ & $285.2 \pm 1266.4$ & $312.8 \pm 656.0$ & $<0.0001$ \\
\hline AST $(\mathrm{IU} / \mathrm{L})$ & $44.4 \pm 48.1$ & $56.3 \pm 56.5$ & 0.0005 \\
\hline Total Bilirubin (mg/dL) & $1.7 \pm 2.1$ & $2.9 \pm 4.2$ & $<0.0001$ \\
\hline Albumin (g/dL) & $3.6 \pm 0.63$ & $3.2 \pm 0.62$ & $<0.0001$ \\
\hline Platelets (x109/L) & $116.0 \pm 62.0$ & $136.4 \pm 81.9$ & 0.0018 \\
\hline $\begin{array}{l}\text { • Wilcoxon rank-sum (Mann-Whitney) test. Abbreviations: Plt, Platelet count; Hb, Haemoglobin; GGTP, Gamma glutamyl transpeptidae; ALKP, Alkaline phosphatase; } \\
\text { AST, Aspartate Aminotransaminase. }\end{array}$ & \\
\hline
\end{tabular}

Table 3: Comparisons in HCC patients between Aggressiveness Index Score of Platelets, GGTP, ALKP, AST, Total Bilirubin and Albumin, in the total cohort.

The mean values were all statistically higher for group the worst Index group than for the lower group in the Index, with respect to GGTP, ALKP, AST, total bilirubin, and platelets, only albumin had a slightly lower value (an index of liver fibrosis) [8,9]. Secondly, a multiple logistic regression model was calculated for the Aggressiveness Index on the liver parameters, each divided into terciles (Table 4).

\begin{tabular}{|c|c|c|c|c|c|}
\hline & OR & $\operatorname{se}(O R)$ & $\mathbf{p}$ & 95\% C.I. & $p \S$ \\
\hline GGTP (IU/mL) & & & & & 0.001 \\
\hline$\leq 33.0$ [Ref. category] & 1 & & & & \\
\hline$>33.0 / \leq 73.2$ & 1.98 & 0.44 & 0.002 & 1.29 to 3.06 & \\
\hline$>73.2$ & 2.09 & 0.47 & 0.001 & 1.34 to 3.26 & \\
\hline ALKP (IU/mL) & & & & & 0.07 \\
\hline$\leq 186$ [Ref. category] & 1 & & & & \\
\hline$>186 / \leq 232$ & 0.84 & 0.18 & 0.42 & 0.54 to 1.29 & \\
\hline$>232$ & 1.41 & 0.28 & 0.08 & 0.96 to 2.08 & \\
\hline AST (IU/L) & & & & & 0.05 \\
\hline$\leq 26.0$ [Ref. category] & 1 & & & & \\
\hline
\end{tabular}


Page 4 of 6

\begin{tabular}{|c|c|c|c|c|c|}
\hline$>26.0 / \leq 44.8$ & 1.07 & 0.23 & 0.77 & 0.70 to 1.63 & \\
\hline$>44.8$ & 1.46 & 0.3 & 0.06 & 0.98 to 2.19 & \\
\hline Total Bilirubin (mg/dL) & & & & & $<0.001$ \\
\hline$<1.0$ [Ref. category] & 1 & & & & \\
\hline$\geq 1.0 / \leq 2.0$ & 1.52 & 0.33 & 0.06 & 0.99 to 2.33 & \\
\hline$>2.0$ & 2.36 & 0.58 & 0.001 & 1.45 to 3.82 & \\
\hline Albumin (g/dL) & & & & & $<0.001$ \\
\hline$<3.5$ [Ref. category] & 1 & & & & \\
\hline$\geq 3.5$ & 0.46 & 0.08 & $<0.001$ & 0.32 to 0.65 & \\
\hline Platelets (×109/L) & & & & & 0.001 \\
\hline$<100$ [Ref. category] & 1 & & & & \\
\hline$\geq 100 / \leq 300$ & 1.55 & 0.27 & 0.01 & 1.11 to 2.18 & \\
\hline$>300$ & 4.98 & 2.3 & 0.001 & 2.01 to 12.30 & \\
\hline
\end{tabular}

Table 4: Multiple logistic regression model of Aggressiveness Index score $(\leq 7 / \geq 8)$, on variables as terciles included together in the model in the total cohort.

The highest Odds Ratios (ORs) were for GGTP, total bilirubin and platelets, and each parameter had a significant p-value for trend of the terciles. Albumin was dichotomized at $<3.5$ or $>3.5 \mathrm{~g} / \mathrm{dl}$, as in the Glasgow score and showed a significant protective effect.

\section{Discussion}

Tumor factors and liver factors have separately and together been acknowledged to be important for HCC prognosis and management decisions. As a result, elements of both sets of factors are included in most HCC classification and prognostication systems [10] with increasing refinements [11]. The underlying liver has been increasingly understood as a complex dynamic of cells, tissues, inflammatory cytokines and growth factors [12-18]. This has been most extensively studied with respect to the neo vascular endothelial cells that form the blood vessels that are needed to support the growing tumor mass, and are an important target for various multikinase inhibitors, which are used in cancer therapy. In addition, several cell types in the tumor microenvironment and their secreted products, are involved in the immune and inflammatory pathways $[13,14]$. Two general categories of inflammation can be discerned in cancer in general, including HCC. They are: (a), local, micro environmental inflammation $[17,18]$, such as that associated with chronic hepatitis [19]; and (b), systemic inflammation, as measured by blood C-reactive protein and albumin levels in the Glasgow and similar inflammation-based prognostic scoring systems $[4-6,20]$. Some micro environmental cell types have multiple functions, such as platelets, which are involved in blood coagulation, metastasis [21], and hepatitis B immune modulation [22], HCC cell growth and migration [23] and the production of HCC growth stimulants [24].

Recently, there has been a paradigm shift, as it has become apparent that the liver microenvironment and liver disease factors are not only independent negative prognostic factors for HCC survival, but may actually be involved in HCC biology. It has long been accepted that growing HCC tumor masses can destroy or replay liver parenchyma and thus enhance liver damage. There is now evidence that the converse is also likely true and that liver micro environmental factors may influence HCC growth and invasion [12-19]. Molecular signatures of the underlying liver in HCC patients provide support for this idea [25-27], as well as recently reported clinical associations of changes in liver function with HCC biology [3].

The experiments reported here had 2 goals. Firstly, to confirm using a different and larger HCC cohort, that the recently-described HCC Aggressiveness Index could be reproduced, with significant survival differences for patients in its 3 score groups of $\mathrm{a}, \mathrm{b}$ and $\mathrm{c}$ (Figure 1). To our knowledge, there has not previously been an HCC index that takes into account all 4 of the commonly-used modalities of HCC human biology, namely tumor size (MTD), tumor multifocality, and portal venous invasion by tumor (PVT) and blood AFP levels. It was expected that the worst category group (highest points) would have the worst survival, and vice versa. We found a decrease in survival with increase in score for each of years 1, 2 and 3 (Figure 1, Kaplan-Meier graph and its associated table). The Cox regression model (Table 2) showed an increasing Hazard Ratio (HR) for death in groups a, b and c, of 1 (reference), 1.94 and 6.64, respectively. The second goal was to examine any possible relationship between the Aggressiveness Index groups and parameters of liver function (Table 3 and multiple logistic regressions in Table 4). We found (Table 3) significantly higher liver parameter values for Aggressiveness group c (Score $>8$ ) compared to Aggressiveness groups a plus b (Score $<8$ ), with respect to GGTP, ALKP, AST and total bilirubin, as well as for platelets (a surrogate for liver cirrhosis $[8,9]$ ). The converse was found for albumin, as expected from its published protective association for survival [4,5]. A multiple logistic regression model for these same parameters as terciles, on the Aggressiveness Index score (Table 4), showed the highest Odds Ratios (OR) were for GGTP, total bilirubin and platelet counts (highest, OR). 
The results presented here show an association between liver function parameters and increasing tumor aggressiveness, but do not show a causal relationship. Nevertheless, the association is quite remarkable. Given the newly available and effective therapies for hepatitis B and C (and possibly also anti-inflammatory agent, such as aspirin, NSAIDS, Cox inhibitors, in patients without bleeding disorder), it may be that their use in suppressing hepatic inflammation and/or improving liver function in HCC patients with inflamed livers, offer the possibility of decreasing HCC aggressiveness through improvement of the underlying liver function [28-32]. What might be the mechanisms by which liver function parameters could influence HCC aggressiveness Two possible general explanations might be considered. A first is that the liver function parameters are only passive measures that reflect the underlying hepato carcinogenic processes of cirrhosis. A second possibility is that the liver parameters actually have HCC-modifying functions. Thus, albumin has been shown to subdue HCC cell growth [33]; HCC-specific GGT isoforms are membrane glycoproteins that catalyze the first enzymatic step in the metabolism of glutathione and is important in maintaining intracellular cysteine and glutathione levels [34-36], ALKP might modulate cancer drug resistance [37] as might bilirubin [38,39]. Furthermore, platelet factors have HCC-stimulatory properties [24].

\section{References}

1. Carr BI, Guerra V (2016) A Hepatocellular Carcinoma Aggressiveness Index and Its Relationship to Liver Enzyme Levels. Oncology 90: 215-220.

2. Schutte K, Bornschein J, Malfertheiner P (2009) Hepatocellular carcinoma - epidemiological trends and risk factors. Dig Dis 27: 80-92.

3. Carr BI, Guerra V, Giannini EG, Farinati F, Ciccarese F, et al. (2014) Association of abnormal plasma bilirubin with aggressive hepatocellular carcinoma phenotype. Semin Oncol 41: 252-258.

4. McMillan DC (2008) An inflammation-based prognostic score and its role in the nutrition-based management of patients with cancer. Proc Nutr Soc 67: 257-262.

5. Pinato DJ, Stebbing J, Ishizuka M, Khan SA, Wasan HS, et al. (2012) A novel and validated prognostic index in hepatocellular carcinoma: the inflammation based index (IBI). J Hepatol 57: 1013-1020.

6. Ishizuka M, Kubota K, Kita J, Shimoda M, Kato M, et al. (2011) Usefulness of a modified inflammation-based prognostic system for predicting postoperative mortality of patients undergoing surgery for primary hepatocellular carcinoma. J Surg Oncol 103: 801-806.

7. Li X, Chen ZH, Xing YF, Wang TT, Wu DH, et al. (2015) Platelet-tolymphocyte ratio acts as a prognostic factor for patients with advanced hepatocellular carcinoma. Tumour Biol 36: 2263-2269

8. Calès P, Oberti F, Michalak S, Hubert-Fouchard I, Rousselet MC, et al. (2005) A novel panel of blood markers to assess the degree of liver fibrosis. Hepatology 42: 1373-1381.

9. Lackner C, Struber G, Liegl B, Leibl S, Ofner P, et al. (2005) Comparison and validation of simple noninvasive tests for prediction of fibrosis in chronic hepatitis C. Hepatology 41: 1376-1382.

10. Sirivatanauksorn Y, Tovikkai C (2011) Comparison of staging systems of hepatocellular carcinoma. HPB Surg 2011: 818217.

11. Chan AW, Kumada T, Toyoda H, Tada T, Chong CC, et al. (2016) Integration of albumin-bilirubin (ALBI) score into Barcelona clinic liver cancer (BCLC) system for hepatocellular carcinoma. J Gastroenterol Hepatol.

12. Tu T, Budzinska MA, Maczurek AE, Cheng R, Di Bartolomeo A, et al. (2014) Novel aspects of the liver microenvironment in hepatocellular carcinoma pathogenesis and development. Int J Mol Sci 15: 9422-9458.

13. Capece D, Fischietti M, Verzella D, Gaggiano A, Cicciarelli G, et al. (2013) The inflammatory microenvironment in hepatocellular carcinoma: a pivotal role for tumor-associated macrophages. Biomed Res Int 2013: 187204.
14. Budhu A, Wang XW (2006) The role of cytokines in hepatocellular carcinoma. J Leukoc Biol 80: 1197-1213.

15. Hernandez-Gea V, Toffanin S, Friedman SL, Llovet JM (2013) Role of the microenvironment in the pathogenesis and treatment of hepatocellular carcinoma. Gastroenterology 144: 512-527.

16. Wu SD, Ma YS, Fang Y, Liu LL, Fu D, et al. (2012) Role of the microenvironment in hepatocellular carcinoma development and progression. Cancer Treat Rev 38: 218-225.

17. Pancoska P, Carr BI (2014) Macro- and micro-environmental factors in clinical hepatocellular cancer. Semin Oncol 41: 185-194.

18. Carr BI, Guerra V (2013) HCC and its microenvironment. Hepatogastroenterology 60: 1433-1437.

19. Bishayee A (2014) The role of inflammation and liver cancer. Adv Exp Med Biol 816: 401-435.

20. Kinoshita A, Onoda H, Imai N, Iwaku A, Oishi M, et al. (2013) The Glasgow Prognostic Score, an inflammation based prognostic score, predicts survival in patients with hepatocellular carcinoma. BMC Cancer 13: 52.

21. Xue TC, Ge NL, Xu X, Le F, Zhang BH, et al. (2016) High platelet counts increase metastatic risk in huge hepatocellular carcinoma undergoing transarterial chemoembolization. Hepatol Res.

22. Sitia G, Iannacone M, Guidotti LG (2013) Anti-platelet therapy in the prevention of hepatitis B virus-associated hepatocellular carcinoma. J Hepatol 59: 1135-1138.

23. Carr BI, Guerra V (2013) Hepatocellular carcinoma size: platelets, $\hat{\mathrm{I}}^{3}$ glutamyl transpeptidase, and alkaline phosphatase. Oncology 85: 153-159.

24. Carr BI, Cavallini A, D'Alessandro R, Refolo MG, Lippolis C, et al. (2014) Platelet extracts induce growth, migration and invasion in human hepatocellular carcinoma in vitro. BMC Cancer 14: 43.

25. Budhu A, Forgues M, Ye QH, Jia HL, He P, et al. (2006) Prediction of venous metastases, recurrence, and prognosis in hepatocellular carcinoma based on a unique immune response signature of the liver microenvironment. Cancer cell 10: 99-111.

26. Hoshida Y, Villanueva A, Kobayashi M, Peix J, Chiang DY, et al. (2008) Gene expression in fixed tissues and outcome in hepatocellular carcinoma. N Engl J Med 359: 1995-2004.

27. Utsunomiya T, Shimada M, Imura S, Morine Y, Ikemoto T, et al. (2010) Molecular signatures of noncancerous liver tissue can predict the risk for late recurrence of hepatocellular carcinoma. J Gastroenterol 45: 146-152.

28. Li X, Liu C, Ip BC, Hu KQ, Smith DE, et al. (2015) Tumor progression locus 2 ablation suppressed hepatocellular carcinoma development by inhibiting hepatic inflammation and steatosis in mice. J Exp Clin Cancer Res 34: 138.

29. Sahasrabuddhe VV, Gunja MZ, Graubard BI, Trabert B, Schwartz LM, et al. (2012) Nonsteroidal anti-inflammatory drug use, chronic liver disease, and hepatocellular carcinoma. J Natl Cancer Inst 104: 1808-1814.

30. Chu TH, Chan HH, Kuo HM, Liu LF, Hu TH, et al. (2014) Celecoxib suppresses hepatoma stemness and progression by up-regulating PTEN. Oncotarget 5: 1475-1490.

31. Zhang H, Zhou Y, Yuan G, Zhou G, Yang D, et al. (2015) Antiviral therapy improves the survival rate and decreases recurrences and fatalities in liver cancer patients following curative resection: A metaanalysis. Mol Clin Oncol 3: 1239-1247.

32. Lee TY, Lin JT, Zeng YS, Chen YJ, Wu MS, et al. (2015) Association between nucleos(t)ide analogue and tumor recurrence in HBV-related hepatocellular carcinoma after radiofrequency ablation. Hepatology.

33. Nojiri S, Joh T (2014) Albumin suppresses human hepatocellular carcinoma proliferation and the cell cycle. Int J Mol Sci 15: 5163-5174.

34. Hanigan MH, Gallagher HC, Townsend DM, Gabarra V (1999) Gammaglutamyl transpeptidase accelerates tumor growth and increases the resistance of tumors to cisplatin in vivo. Carcinogenesis 20: 553-559.

35. Wang NY, Zhang D, Zhao W, Duan MH, et al. (2009) Clinical application of an enzyme-linked immunosorbent assay detecting hepatoma-specific gamma-glutamyl transferase. Hepatol Res 39: 979-987. 
Citation: Carr BI, Guerra V, Giannini EG, Farinati F, Ciccarese F, et al. (2016) An HCC Aggressiveness Index and Blood GTP, Bilirubin and Platelet Levels. J Integr Oncol 5: 172. doi:10.4172/2329-6771.1000172

Page 6 of 6

36. Franzini M, Corti A, Lorenzini E, Paolicchi A, Pompella A, et al. (2006) Modulation of cell growth and cisplatin sensitivity by membrane gammaglutamyltransferase in melanoma cells. Eur J Cancer 42: 2623-2630.

37. Efferth T, Volm M (1993) Increase of alkaline phosphatase in multidrugresistant tumor cells and their cross-resistance to 6-thioguanine. Arzneimittelforschung 43: 1118-1121.

38. Nomura M, Matsunami T, Kobayashi K, Uchibayashi T, Koshida K, et al (2005) Involvement of ABC transporters in chemosensitivity of human renal cell carcinoma, and regulation of MRP2 expression by conjugated bilirubin. Anticancer Res 25: 2729-2735.

39. Tamai M, Ito Y, Mirura H, Tsukada H, Yokogawa K, et al. (2003) Conjugated bilirubin induces multidrug resistance-associated protein 2 mRNA expression and in vivo cisplatin resistance in rat hepatoma AH66 cells. Anticancer Res 23: 4781-4787. 